\title{
Sexualbezogene Online-Fortbildung für Fachkräfte: Webinare und andere E-Learning-Kurse
}

\author{
Nicola Döring ${ }^{\mathrm{a}}$
}

Zusammenfassung: Der vorliegende Praxisbeitrag stellt insgesamt sieben sexualwissenschaftlich fundierte E-Learning-Angebote von und für Fachkräfte vor. Darunter befinden sich vor allem Webinare, aber auch andere E-Learning-Formate. Thematisch wird ein weiter Bogen gespannt von sexueller Gesundheit über Transphobie und Trans*Identitäten, Sexualitäten bei Menschen mit Demenz, genitaler Stimulation und Orgasmus bei Frauen bis hin zu Techniken der Sexual- und Paartherapie.

Schlüsselwörter: Internet; Online-Fort- und Weiterbildung; Sexualtherapie; sexuelle Gesundheit; Transgender

\section{Sex-Related Online Training for Professionals: Webinars and Other E-Learning Courses}

\begin{abstract}
This practice-oriented article presents a total of seven e-learning courses based on sexual science from and for professionals. Most consist of webinars, but there are also other e-learning formats. The topics range from sexual health, transphobia and trans*identities, sexualities in people with dementia, genital stimulation and orgasm in women to techniques of sexual and couple therapy.
\end{abstract}

Key Words: internet; online professional training; sexual health; sexual therapy; transgender

Für die informelle sexualbezogene Online-Fortbildung von Fachkräften stehen im Internet diverse Ressourcen zur Verfügung (Döring 2018a). Dazu gehören unter anderem Webvideos auf YouTube (Döring 2017a, 2017b), die im letzten Heft der „Zeitschrift für Sexualforschung“ ausführlich behandelt wurden (Döring 2018b). Im Unterschied zu lehrreichen Webvideos haben die im vorliegenden Beitrag behandelten Webinare (Kofferwort aus Web und Seminar) einen noch stärkeren didaktischen Anspruch: Hier wird nicht

a Institut für Medien- und Kommunikationswissenschaft, Technische Universität Ilmenau

Z Sexualforsch 2018; 31; 295-304

(c) Georg Thieme Verlag KG Stuttgart · New York

ISSN 0932-8114

DOI 10.1055/a-0664-4924 
nur auf das gesprochene Wort gesetzt, sondern es werden auch Präsentationsfolien, Quizfragen und in Live-Webinaren sogar Gruppenarbeitsphasen integriert (Luber und Geisler 2016). Voraufgezeichnete sowie live durchgeführte Webinare sind eine wichtige Form des zeitgenössischen E-Learnings. Daneben existieren weitere Formate wie z.B. E-Learning-Ressourcen in Form von digitalen Broschüren oder Online-Kurse in Form von MOOCs (Massive Open Online Courses).

Der vorliegende Beitrag stellt insgesamt sieben sexualwissenschaftlich fundierte E-Learning-Angebote von und für Fachkräfte vor. Darunter befinden sich vor allem Webinare, aber auch eine E-Learning-Ressource in Form einer digitalen Broschüre sowie ein MOOC. In die Auswahl einbezogen wurden ausschließlich Angebote, die von sexualwissenschaftlich qualifizierten Fachkräften stammen, in deutscher und/oder englischer Sprache vorliegen und Nachhaltigkeit zeigen. Bevorzugt wurden zudem kostenlose Angebote sowie Angebote, zu denen bereits Evaluationsergebnisse vorliegen. Thematisch wird ein weiter Bogen gespannt von sexueller Gesundheit über Transphobie und Trans*Identitäten, Sexualitäten bei Menschen mit Demenz, genitaler Stimulation und Orgasmus bei Frauen bis hin zu Techniken der Sexual- und Paartherapie.

\section{E-Learning-Studiengang „Sexuelle Gesundheit“}

Der deutsche Sexualwissenschaftler Erwin J. Häberle gründete im Jahr 1994 am Robert-Koch-Institut das Archiv für Sexualwissenschaft, basierend auf Werken aus seiner privaten Bibliothek. Der elektronische Teil des Archivs steht heute als Archive for Sexology auf dem von Erwin Häberle privat betriebenen und durch Spenden unterstützten Webserver Sexarchive.info (http:// www.sexarchive.info/) kostenlos zur Verfügung und präsentiert Inhalte in 15 Sprachen. Sexarchive.info bietet Informationen über die Geschichte der Sexualforschung, präsentiert wichtige Studien und Publikationen im Volltext, enthält auch ein von Häberle verfasstes Glossar zentraler Fachbegriffe. Verlinkt wird zudem auf die Website „The Sexology Expert“ (https://sexologyexpert.online/), auf der Häberle sein Wissen in Form von kurzen Videos teilt: Es finden sich rund 20 Webinare zu Themen wie Verhütung, Homosexualität und weibliche Genitalverstümmelung.

Teil von Sexarchive.info ist zudem der E-Learning-Studiengang „Sexuelle Gesundheit“ (http://www.sexarchive.info/Home_DE/Startseite/E_LearningKurse/e_learning-kurse.html), der auf Deutsch und Englisch sowie in weiteren Sprachen vorliegt.

Ziel des E-Learning-Studiengangs „Sexuelle Gesundheit“ ist es, sexualwissenschaftliches Grundlagenwissen für Fachpersonal im Bereich Gesundheit und Bildung zu vermitteln. Angelegt ist das zwischen 2003 und 2013 entwickelte Kursprogramm auf sechs Online-Lektionen, von denen jedoch nur die ersten vier vorliegen:

1. Grundlagen der sexuellen Anatomie und Physiologie beim Menschen

2. Die menschliche Fortpflanzung 
3. Körperliche Probleme beim weiblichen und männlichen Geschlecht

4. Sexuell übertragbare Krankheiten

5. Sexuelle Funktionsstörungen und ihre Behandlung (liegt nicht vor)

6. Das menschliche Sexualverhalten (liegt nicht vor)

Jede Online-Lektion besteht aus mehreren Kapiteln, Listen von Ja-/Neinund Multiple-Choice-Examensfragen (ohne Antworten) sowie einigen Literaturangaben. Die Aufbereitung der Inhalte ist textbasiert mit vereinzelten Abbildungen und bleibt somit - in den Worten des Entwicklers - „ohne alle technischen Mätzchen“. Gedacht sind die Inhalte sowohl zum Selbstlernen als auch zur Integration in den Präsenzunterricht.

Der E-Learning-Studiengang wird laut Angaben auf der Website von zahlreichen sexualwissenschaftlichen Fachorganisationen empfohlen und verzeichnet „Millionen von Nutzenden in über 200 Ländern“, was das Angebot zum „größten E-Learning-Projekt der Welt“ mache (http://www. sexarchive.info/Entrance_Page/Free_Online_Courses/free_online_courses. html). Aktuelle Webstatistiken von Sexarchive.info verzeichnen indessen so geringe Abrufzahlen, dass eine genaue Auswertung gar nicht möglich ist (https://www.similarweb.com/website/sexarchive.info).

Sein unermüdlicher Einsatz für die internationale Verbreitung sexualwissenschaftlicher Kenntnisse ist Erwin Häberle hoch anzurechnen. Gleichzeitig sind die Grenzen spürbar, die er als engagierte Einzelperson bei einem so großen Projekt erlebt: Bei der Durchsicht der Online-Kurse fallen sofort das veraltete Webdesign und die toten Links auf. Inhaltlich mag man sich - je nach fachlichem Hintergrund - bei den vermittelten Informationen andere Akzentsetzungen, modernere Formulierungen und auch einen engeren Anschluss an den aktuellen Forschungsstand wünschen. So finden sich in der ersten Lektion zum Thema „Die sexuelle Reaktion - Die weibliche Reaktion“ beispielsweise Sätze wie diese: „Die moderne Sexualforschung hat gezeigt, dass die sexuelle Reaktion bei beiden Geschlechtern demselben Grundmuster folgt. In der Tat sind die weiblichen sexuellen Fähigkeiten den männlichen mindestens gleichwertig, teilweise sogar überlegen“ (http://www.sexarchive.info/ECD1/weibliche_reaktion.html). Dass hier von nur zwei Geschlechtern ausgegangen wird, dass normative Bewertungsmaßstäbe an sexuelle Fähigkeiten angelegt werden und zudem suggeriert wird, es gäbe wissenschaftlichen Konsens hinsichtlich eines geschlechterübergreifenden „Grundmusters“ in der sexuellen Reaktion, wirkt unzeitgemäß.

Bei allen Kritikpunkten bleibt jedoch festzuhalten, dass das Projekt eines der ganz wenigen Beispiele für den Versuch darstellt, sexualwissenschaftliche Grundlagen in Online-Kursen kostenlos und mehrsprachig zu verbreiten. 


\section{Webinar „Transgender Awareness“}

Die US-amerikanische klinische Psychologin Lauren Mizock (www. laurenmizock.com) arbeitet unter anderem in der Versorgung von Trans*Personen und forscht auch auf diesem Gebiet. Vor dem Hintergrund ihrer praktischen Erfahrungen und empirischen Befunde zu Transphobie hat sie im Jahr 2014 das englischsprachige „Transgender Awareness Webinar“ entwickelt, das über Trans*Themen aufklären und Transphobie reduzieren will. Der Begriff „Transphobie“ meint negative Reaktionen und Einstellungen sowie diskriminierendes Verhalten gegenüber Trans*Personen.

Das Webinar steht online frei zur Verfügung (https://sites.google.com/ site/laurenmizockphd/transgender-webinar). Es existiert in zwei Fassungen: Version 1 ist für die breite Öffentlichkeit ausgelegt und Version 2 (in Zusammenarbeit mit Ruben Hopwood) für Fachkräfte aus dem Gesundheitswesen. Jede Webinar-Version besteht aus einem integrierten Satz an Informationsfolien, Grafiken, Videos und diversen Multiple-Choice-Tests, wobei die Bearbeitung insgesamt etwa 60 Minuten Zeit beansprucht. Die Webinar-Variante für Fachleute geht neben Begriffsklärungen stärker auch auf Theorien (z. B. zur Entwicklung geschlechtlicher Identität oder zu Wirkungen von Transphobie) ein und thematisiert zudem die verschiedenen Formen von Unterstützung, die Fachkräfte Trans*Personen geben können. Auch typische Formen der (teilweise unbewussten) Diskriminierung von Trans*Personen (z.B. durch unangemessene Fragen oder Verwendung falscher Personalpronomen) werden thematisiert.

Zusammen mit Kolleg_innen hat die Entwicklerin Lauren Mizock beide Webinar-Fassungen in einem Prä-Post-Design evaluiert und die Befunde publiziert (Mizock et al. 2017). Dabei zeigte sich an einer Stichprobe von $\mathrm{N}=303$ Studierenden und einer Stichprobe von N=158 Fachkräften aus dem Gesundheitswesen, dass transphobische Einstellungen nach dem Kurs signifikant geringer waren als vorher, wobei sich mittlere $(d=-0.75)$ bis große $(\mathrm{d}=-1.01)$ Effektstärken zeigten. Im Vergleich mit Anti-Stigma-Trainings, die face-to-face durchgeführt werden, hat die Webinar-Variante den Vorteil, dass sie orts- und zeitflexibel einsetzbar ist. Indem Trans*Personen und Trans*Fachleute im Webinar per Video zu Wort kommen, wirkt das OnlineTraining authentisch. Im Vergleich zu den diversen Webvideos auf YouTube, die sich Trans*Themen widmen (Döring 2018b), ist das Webinar wesentlich stärker durchstrukturiert und didaktisch aufbereitet.

\section{E-Learning-Ressource „Sexualities and Dementia“}

Die australische Psychologin und Gerontologin Cindy Jones beschäftigt sich in Forschung und Praxis unter anderem mit den Sexualitäten von Menschen mit Demenz-Erkrankungen. Vor dem Hintergrund, dass erwünschte Sexualität für viele Menschen - einschließlich Menschen mit Demenz zu Gesundheit und Lebensqualität beiträgt und dass alle Menschen - einschließlich Menschen mit Demenz - sexuelle Rechte haben, hat sie die 
E-Learning-Ressource „Sexualities and Dementia“ für Fachleute im Gesundheitswesen entwickelt (https://www.privacy.org.nz/assets/Uploads/ Jones-C.-2014.-Sexualities-and-Dementia-Education-Resource-for-HealthProfessionals-Manual2.pdf).

Das englischsprachige Lernmaterial besteht aus einer 70-seitigen farbigen digitalen Broschüre, die Texte, Bilder, Fallbeispiele, Verweise auf OnlineVideos und Quiz-Fragen enthält sowie einen umfangreichen Anhang mit Instrumenten zur Messung und Klassifikation des einvernehmlichen sowie des grenzverletzenden Sexualverhaltens von Menschen mit Demenz. Das Material kann zum Selbstlernen genutzt werden, dient aber auch als Basis für Präsenz-Workshops und Webinare, die von Cindy Jones angeboten werden.

Die E-Learning-Ressource ist in vier Module aufgeteilt:

- Modul A: „Intimacy, Sexuality, and Sexual Behavior“

- Modul B: „Dementia and the Expression of Sexuality“

- Modul C: „Ethical Considerations: Policy / Guidelines Development for Sexualities and Dementia in Care Settings“"

- Modul D: „Developing Sexualities and Dementia Policy Guidelines for Care Practice“

Die E-Learning-Ressource möchte Fachleute im Gesundheitsbereich für die Sexualitäten von Menschen mit Demenz sensibilisieren und darauf hinwirken, dass Pflegeeinrichtungen Konzepte zum professionellen und rechtebasierten Umgang mit Sexualität und Demenz in Pflegeeinrichtungen entwickeln und implementieren.

Eine Evaluationsstudie mit $\mathrm{N}=42$ Teilnehmenden aus der Zielgruppe zeigte in einem Prä-Post-Design, dass das Wissen über die Sexualitäten von Menschen mit Demenz signifikant anstieg, während negative Einstellungen gegenüber sexuellem Ausdruck von Menschen mit Demenz signifikant zurückgingen (Jones und Moyle 2016). Besonders komplexe Fragen sind dabei die sexuelle Konsensfähigkeit von Menschen mit Demenz und die angemessene Gesprächsführung zwischen Einrichtungspersonal und Angehörigen hinsichtlich sexueller Aktivitäten der Bewohner_innen. Die Grenze des Selbstlernmaterials liegt naturgemäß darin, dass ein interaktiver Austausch mit anderen Lernenden sowie mit der Lehrkraft fehlt.

\section{E-Learning-Plattform OMGyes}

Die im Dezember 2015 gelaunchte E-Learning-Plattform OMGyes (www. OMGyes.com) ist benannt nach dem lustvollen Ausruf „Oh My God, yes“. Sie ist in englischer und deutscher Sprachversion verfügbar. OMGyes hat sich zum Ziel gesetzt, wissenschaftsbasiert und gleichzeitig sehr praxisnah über die vielfältigen Muster sexueller Lust, genitaler Berührung und Orgasmen bei Frauen aufzuklären. Betrieben wir die Website von dem US-Unternehmen For Godness Sake LLC (https://www.fgsake.org/), die führenden Köpfe sind die Fotografin Lydia Daniller und der Webberater Rob Perkins. Koope- 
rationspartnerin in der Forschung ist die US-amerikanische Sexualwissenschaftlerin Debbie Herbenick, Direktorin des Center for Sexual Health Promotion an der Indiana University in Bloomington. Sie führte 2015 eine von OMGyes finanzierte bevölkerungsrepräsentative Umfrage in den USA unter $\mathrm{N}=1055$ Frauen zwischen 18 und 94 Jahren durch. Die befragten Frauen gaben detailliert Auskunft über die von ihnen beim Solo- und Partnersex praktizierten und bevorzugten körperlichen Stimulationsarten (Herbenick et al. 2018).

Die wissenschaftlichen Befunde dieser ersten Studie wurden zu zwölf Techniken verdichtet, die Lust und Orgasmen verschaffen können, etwa „Hochschaukeln“, „Umspielen“, „Dranbleiben“, „Umkreisen“ oder „Abfedern“. Jede Technik wird mit Texten und Grafiken erklärt. Zudem werden sie in authentischen Video-Erfahrungsberichten von Frauen unterschiedlicher Altersgruppen, Hautfarben und Körpertypen beschrieben und vor der Kamera demonstriert. Die Frauen liegen dabei entspannt auf dem Bett, erklären und zeigen zwischen geöffneten nackten Schenkeln, wie schnell oder langsam, wie fest oder sanft, in welche Richtungen und an welchen Stellen sie sich genital berühren. Zusätzlich können die demonstrierten Techniken an interaktiven Simulationen erprobt werden. Dabei sind die Muster der genitalen Stimulation mit der Maus nachzuvollziehen, wobei das System akustisches Feedback gibt: Man wird also etwa fordernd oder schmeichelnd zu schnelleren oder langsameren Bewegungen aufgefordert, hört wohliges Stöhnen, schnelleres Atmen.

Die inhaltliche und mediale Aufbereitung der Plattform ist hochprofessionell und hat ein Budget von mehreren Millionen US-Dollar erfordert. Finanziert wird das Angebot über Mitgliedsbeiträge, die aktuell bei einer Einmalzahlung von 39 Euro liegen. Fachleute im Bereich sexuelle Gesundheit und Sexualaufklärung können das Material nutzen, um mit ihren Zielgruppen zu arbeiten. Laut aktuellen Webstatistiken verzeichnet OMGyes knapp eine halbe Million Besucher_innen pro Monat (https://www.similarweb.com/ website/omgyes.com), wobei die meisten aus Deutschland und den USA stammen. Durch intensives Marketing, Testimonials und positive Presseresonanz ist die Plattform bereits recht bekannt. Mitglieder der International Academy of Sex Research (IASR) können einen kostenlosen Testzugang beantragen, indem sie eine E-Mail an alecia@omgyes.com senden und in die Betreffzeile „IASR Member“ schreiben.

Evaluationsstudien der Plattform stehen aus. Dass die vorliegende erste Staffel des Angebotes den Fokus auf die Mikroebene der genitalen Stimulation richtet, mag für gespaltene Einschätzungen sorgen: Manchen wird das E-Learning-Angebot, das unter anderem der Frage nachgeht, ob Stimulation links oder rechts der Klitorisspitze angenehmer ist, wohl etwas zu mechanistisch vorkommen. Andere werden es zu schätzen wissen, dass das vielbeschworene Mantra „Der Orgasmus ist bei jeder Frau anders“ hier einmal ernst genommen wird und die diversen Varianten der Stimulation ganz kleinteilig und anschaulich durchdekliniert werden. Dass dafür auch bei bester medialer Aufbereitung Geduld notwendig ist, liegt in der Natur der Sache. Was OMGyes zusammen mit den handfesten Techniken vermittelt, ist 
das präzise und hilfreiche Benennen und Besprechen derselben, wobei die authentischen Videos sehr sympathische Rollenmodelle bieten.

\section{Webinar-Reihe „The Sex Experts“}

Das Center for Healthy Sex (CHS) (http://centerforhealthysex.com/) in Los Angeles ist laut Selbstbeschreibung eine der führenden Kliniken für Sex-, Pornografie- und Liebessucht in den USA. Angeboten werden Einzel- und Paartherapien sowie Fortbildungen für Sexualtherapeut_innen. Gegründet und geleitet wird das CHS von der klinischen Psychologin und Sexualtherapeutin Alexandra Katehakis, die sich für eine starke Präsenz der Klinik in Sozialen Medien einsetzt. Die Klinik veröffentlicht regelmäßig Webinare sowohl für die breite Bevölkerung als auch für Fachleute. Dazu gehört die „Sex Experts Webinar Series“, in deren Rahmen seit 2014 jeden Monat ein Webinar über den YouTube-Kanal der Klinik hochgeladen wird (https:// www.youtube.com/user/CenterForHealthySex/playlists).

Bislang liegen mehr als vierzig jeweils rund einstündige Sex-ExpertWebinare vor, etwa zu Themen wie „Understanding and using your client's sexual fantasies in therapy“ (Piper Grant: https://www.youtube. com/watch?v=HnJrZXghpEk), „Bearing witness: therapeutic support for the coming-out process" (Dan Lacovara: https://www.youtube.com/ watch?v=J-y5 msTDyc8) oder „Art therapy for survivors of sexual trauma“ (Pamela Hayes: https://www.youtube.com/watch?v=8fW3moIAP98). Die Webinare können Fachleuten niedrigschwellig neue Anregungen für die praktische Arbeit liefern und vorhandene Kenntnisse auffrischen. Ebenso sind über den Kanal mehr als vierzig Fachvorträge zu finden. Alle Inhalte sind kostenlos, da die Videos Marketing-Funktionen sowohl für die Klinik als auch für die referierenden Fachleute erfüllen, die auf ihre Beratungsund Therapie-Angebote sowie ihre Bücher verweisen. Die Beiträge drehen sich um Sex-, Pornografie- und Liebessucht, deren Erscheinungsformen, Ursachen und Behandlungsmöglichkeiten, aber auch um grundlegende Fragen sexueller Gesundheit, Intimität und therapeutische Techniken. Die Webinare und Webvorträge sind einfach produziert, zeigen per Video in der Regel die Präsentationsfolien und die vortragende Person. Zuweilen würde man sich eine bessere Bild- und Tonqualität wünschen, auch liegen nicht für alle Videos englische oder gar deutsche Untertitel vor.

\section{Live-Webinar-Reihe „Fallanalysen-Konferenz“}

Der US-amerikanische klinische Psychologe, Sexual- und Paartherapeut David Schnarch bietet zu seinem Ansatz der Crucible Therapy in den USA (http:// crucibletherapy.com/) und in Deutschland (http://crucibleinstitute.de/) Weiterbildungs-Workshops für Therapeut_innen an. Zusätzlich organisiert er eine monatliche Live-Webinar-Reihe als „Fallanalysen-Konferenz“ (http:// crucibleinstitute.de/crucible-therapie-webinar-serie). Zu jeder Live-Webi- 
nar-Sitzung wird zuvor eine mehrseitige schriftliche Fallbeschreibung aus der Praxis bereitgestellt, über die in der Online-Fallanalysen-Konferenz mit David Schnarch diskutiert wird. Als Plattform wird Adobe Connect verwendet, sodass sich die Teilnehmenden in einem virtuellen Meetings-Raum treffen und schriftlich oder mündlich diskutieren können. Für ein einzelnes Webinar werden Teilnahmegebühren von 75 Euro abgerechnet, im Abonnement sinkt der Preis auf 50 Euro. Für bereits stattgefundene Webinare können zu gleichen Preisen die Video-Aufzeichnungen bezogen werden.

Das Webinar vom April 2018 behandelt den von einer Therapeutin eingereichten Fall eines Patienten, der sie ursprünglich wegen sexueller Probleme aufgesucht hatte, wobei nach mehrjähriger Behandlung nun aber Beziehungsprobleme im Fokus stehen. Der Patient zeigt ein kontrollierendes und teilweise massiv übergriffiges Verhalten gegenüber Partnerinnen und beschwert sich darüber, dass Frauen sich von ihm stets nach kurzer Zeit trennen und zu wenig Empathie für ihn aufbringen würden. In der Webinar-Sitzung sind die Therapeutin, David Schnarch und die Dolmetscherin in drei Video-Fenstern zu sehen, die Teilnehmenden können über ein ChatFenster kommentieren. Gesprochen wird auf Englisch, die Dolmetscherin übersetzt ins Deutsche. David Schnarch geht unter anderem auf die in der schriftlichen Fallbeschreibung dokumentierten Äußerungen und Denkprozesse des Patienten ein, die er als psychotisch kennzeichnet. Er diskutiert die Grenzen der Behandlung eines Patienten, dessen Verhalten strafrechtlich relevant ist, und der seine Aggression möglicherweise auch gegen die Therapeutin richten könnte. Obwohl das Live-Webinar diverse Interaktionsmöglichkeiten bietet, gestaltet sich die Fallanalysen-Konferenz doch im Wesentlichen monologisch, indem Schnarch den Fall kommentiert. Die beschränkte Ton- und Bildqualität sowie die zeitversetzte Übersetzung sind spürbare Einschränkungen des Formats, die jedoch von denjenigen in Kauf genommen werden, die gern eine regelmäßige Fallanalysen-Konferenz mit David Schnarch erleben wollen.

\section{Coursera MOOC „What does it mean to identify as Transgender or Gender Non-Conforming (TGNC)?“}

Unter einem MOOC (Massive Open Online Course) versteht man einen Online-Kurs (meist im Rahmen der Hochschul- oder Erwachsenenbildung), der im Internet niedrigschwellig und kostenlos von großen Menschenmengen absolviert werden kann, einschließlich der Vergabe von Teilnahmezertifikaten bei erfolgreichem Abschluss. Die ursprüngliche Idee bestand darin, Veranstaltungen renommierter Universitäten wie etwa der Stanford University auch Außenstehenden zugänglich zu machen. Der erste große Hype um MOOCs ist zehn Jahre nach der Einführung des Konzepts etwas abgeflaut, denn MOOCs leiden wie alle Fernausbildungsformate an hohen Abbruchraten.

Für die sexualwissenschaftliche Online-Fortbildung von Fachkräften ist das auf der von zwei Stanford-Professoren gegründeten Plattform Coursera 
offerierte MOOC „What does it mean to identify as Transgender or Gender Non-Conforming (TGNC)?" (https://www.coursera.org/learn/tgncgender-identity-social-change/) von Bedeutung. Angeboten wird es von Rebecca Graetz vom „Program in Human Sexuality“ der Universität Minnesota (https://www.sexualhealth.umn.edu/). Eli Coleman, Direktor des Programms, leitet das MOOC mit einer Videobotschaft ein. Angelegt ist das MOOC auf eine Bearbeitungszeit von acht Wochen. Es enthält acht Lektionen mit Lehrvideos und Lehrtexten, Hintergrundmaterialien und MultipleChoice-Tests. Es geht beispielsweise um sexuelle Identitätsentwicklung, Coming-out und Transition, um die Perspektiven von Eltern und Ehepartner_innen, um Diskriminierung im Gesundheitssystem, im Arbeitsleben und in Bildungseinrichtungen, um wohnungslose Trans*Personen und die Situation von Trans*Personen im Gefängnis. Das Material ist englischsprachig, für die Videos existieren Untertitel und Transkripte. Nach einer Registrierung auf der Coursera-Plattform kann das gesamte MOOC jederzeit kostenfrei genutzt werden. Wer jedoch ein Abschlusszertifikat erwerben möchte, muss eine Kursgebühr von 41 Euro entrichten. Genutzt wird das MOOC laut Coursera-Statistik von Lernenden aus den USA, aus Europa und Asien (Graetz 2017). Die Teilnahme vermittelt ein vertieftes Verständnis von Trans*Themen und ist für Fachleute auch deswegen nützlich, weil sie über das MOOC auf einen großen Pool handverlesener, öffentlich zugänglicher Quellen aufmerksam werden (YouTube-Videos, Zeitungsartikel, Aufklärungsmaterialien, Guidelines usw.), die sie selbst mit und für ihre Klient_innen nutzen können.

\section{Fazit}

Technisch und inhaltlich hochwertige Webinare und andere E-LearningAngebote für die sexualbezogene Fortbildung von Fachkräften sind bislang im deutschsprachigen sowie im internationalen Raum noch recht rar zumindest wenn es um niedrigschwellige, d.h. um gut auffindbare und kostenlose (oder relativ kostengünstige) Angebote geht. Die hier präsentierte Auswahl von sieben Angeboten zeigt Stärken, aber auch Schwächen dieser Art von Online-Fortbildung auf. Unbestreitbar werden E-Learning-Angebote in Zukunft eine noch wichtigere Rolle im gesamten Spektrum der Fortbildungsformate spielen. Es mehren sich auch die Ansätze, E-Learning-Angebote in der formalisierten Weiterbildung zu verankern. So bietet der ThiemeVerlag diverse, oft aus Zeitschriften-Artikeln abgeleitete Online-Module zum Erwerb von CME-Punkten (https://cme.thieme.de/cme-webapp/), darunter auch Angebote zu sexualbezogenen Themen. Die österreichische Ärztekammer bietet beispielsweise einen Zertifikatslehrgang „Sexualmedizin“ an, der aus Grundkurs, Supervision und E-Learning besteht (https:// www.arztakademie.at/fortbildungsangebot/e-learning/e-learning-sexual medizin/). Vor dem Hintergrund fortschreitender Digitalisierung ist es wünschenswert, dass sich die sexualwissenschaftlichen Fachgesellschaften und andere in die Fortbildung involvierte Institutionen in Deutschland der 
Entwicklung von inhaltlich und medial zeitgemäßen E-Learning-Angeboten verstärkt zuwenden, bei denen es sich - das muss immer wieder betont werden - nie um einen Ersatz, sondern immer um eine sinnvolle Ergänzung von Präsenzunterricht handeln sollte.

\section{Literatur}

Döring N. Online-Sexualaufklärung auf YouTube: Bestandsaufnahme und Handlungsempfehlungen für die Sexualpädagogik. Z Sexualforsch 2017a; 30: 349-367

Döring N. Sexualaufklärung im Internet: Von Dr. Sommer zu Dr. Google. Bundesgesundheitsblatt Gesundheitsforschung Gesundheitsschutz 2017b; 60: 1016-1026

Döring N. Sexualbezogene Online-Fortbildung für Fachkräfte: Eine Einführung. Z Sexualforsch 2018a; 31: 97-100

Döring N. Sexualbezogene Online-Fortbildung für Fachkräfte: Webvideos. Z Sexualforsch 2018b; 31: $97-100$

Graetz R. What Does it Mean to Be Transgender or Gender Non-Conforming? A MOOC on Diversity and Inclusion. Presentation at the Minnesota eLearning Summit. 2. August 2017 [Als OnlineDokument: https://pubs.lib.umn.edu/index.php/mes/issue/view/81]

Herbenick D, Fu TJ, Arter J, Sanders SA, Dodge, B. Women's Experiences with Genital Touching, Sexual Pleasure, and Orgasm: Results from a U.S. Probability Sample of Women Ages 18 to 94 . J Sex Marital Ther 2018; 44: 201-212

Jones C, Moyle W. Sexuality \& Dementia: An E-Learning Resource to Improve Knowledge and Attitudes of Aged Care Staff. Educ Gerontol 2016; 42: 563-571

Luber S, Geisler, I. Online-Trainings und Webinare. Von der Vermarktung bis zur Nachbereitung. Weinheim, Basel: Beltz 2016

Mizock L, Hopwood R, Casey H, Duhammel E, Herrick A, Puerto G, Stelmach J. The Transgender Awareness Webinar: Reducing Transphobia among the General Public and Mental Health Providers. J Gay Lesbian Ment Health 2017; 21: 292-315

Korrespondenzadresse

Prof. Dr. phil. Nicola Döring

Technische Universität Ilmenau

Institut für Medien- und Kommunikationswissenschaft

Ehrenbergstr. 29 (EAZ 2217)

98693 Ilmenau

Nicola.Doering@tu-ilmenau.de 